

\section{The Thalassa Mission Concept}

The Thalassa mission concept was developed for a proposal submitted to the NASA Planetary Mission Concept Studies call in May 2019. In this White Paper, we describe the mission concept, its overarching scientific focus and objectives, and our notional implementation for tackling those objectives. By combining a comprehensive instrument suite with multiplatform architecture-an orbiter, an aerial platform, and dropsondes - the Thalassa mission concept has been designed to explore the upper and middle atmosphere and the surface of Venus, motivated by a single scientific focus: Determine the extent to which water has played a role in the geological evolution of Venus.

\subsection{Introduction}

There is substantial evidence that Venus once possessed much more water than it does today (Treiman, 2007; Elkins-Tanton et al., 2007; Way and Del Genio, 2020), raising the tantalizing prospect that the second planet may have long been Earth-like and even habitable. But whether this water was truly present, when and how it was lost, if geological or geochemical evidence of this water remains preserved on the surface, and even whether Venus and Earth started off with similar water inventories are major, unanswered questions (Smrekar et al., 2007). Establishing the extent to which Venus was once a water world will substantially improve our understanding of this enigmatic planet, of the geological evolution of our own world and the inner Solar System more broadly, and of the rules that govern Earth-size worlds in planetary systems in general. Our mission concept is named for the primordial spirit of the sea in Greek mythology, and traces back to the theme "follow the water." This theme is borrowed from the Mars community, where this approach has yielded enormous dividendseven though oceans on Venus could have outlasted any such features on Mars by billions of years.

\subsection{Science Focus}

The Thalassa scientific focus is arguably the highest-priority goal for Venus exploration, and encompasses climate, geology, and geophysics. Water is the key to Earth's habitability (e.g., Tosi et al., 2017) and likely plays a major role in plate tectonics (Smrekar et al., 2007). Therefore, water is a vital clue to the mystery of whether early Venus and early Earth were similar and, if so, when and why the geological histories of these two planets diverged.

Thalassa's scientific focus is directly traceable to NASA's strategic objectives for planetary science. The 2018 NASA Strategic Plan charged the Science Mission Directorate with "implementing the Decadal Survey priorities." Likewise, the 2014 NASA Science Plan declared that "scientific priorities for future planetary science missions are guided by the recommendations of the decadal surveys." To those ends, Thalassa addresses priority questions from all three broad, crosscutting themes in the 2011 "Vision and Voyages for Planetary Science in the Decade 2013-2022" Decadal Survey:

a) What governed the accretion, supply of water, chemistry, and internal differentiation of the inner planets and the evolution of their atmospheres? ("Building new worlds" theme);

b) Did... Venus host ancient a queous environments conducive to early life? ("Planetary habitats" theme);

c) How have the myriad chemical and physical processes that shaped the Solar System operated, interacted, and evolved over time? ("Workings of solar systems" theme).

The Thalassa scientific focus also directly addresses several high-priority investigations from the 2019 Venus Exploration Analysis Group (VEXAG) report "Scientific Goals, Objectives, and Investigations (GOI) for Venus Exploration." In particular, the investigation of the role(s) of water in Venus' geological history firmly traces to the $2019 \mathrm{GOI}$ Goal I (Understand Venus' early evolution and potential habitability) and its Objective I.A. (Did Venus have temperate surface conditions and liquid water at any time?). Objective I.A., in turn, encompasses investigations including: I.A.HO. Hydrous Origins. Determine 
whether Venus shows evidence for a bundant silicic igneous rocks, I.A.AL. Atmospheric Losses. Quantify the processes by which the atmosphere of Venus loses mass to space; and I.A.MA. Characterize the distribution of any remanent magnetism in the crust of Venus. The Thalassa scientific focus also encompasses $2019 \mathrm{GOI}$ investigations considered "essential," including I.B.IS. (Isotopes), III.A.GC. (Geochemistry), and III.A.GA. (Geologic Activity), as well as II.B.OG. (Outgassing).

Finally, Thalassa is directly responsive to the mission concepts required for the next decadal survey in planetary science. According to the 2017 National Academy of Sciences (NAS) Committee on Astrobiology and Planetary Science (CAPS) report "Getting Ready for the Next Planetary Science Decadal Survey," Venus exploration is a high-priority science area requiring additional studies. A joint U.S.-Russian science definition team recently studied a Flagship-class Venus orbiter and lander concept, Venera-D. However, the NAS CAPS report noted that "some of the scientific approaches to Venus... were previously thought to be possible only on the surface but might be possible from space or a balloon-hosted instrument." Thalassa exploits this new realization, and our proposed combination of orbiter, aerial platform, and dropsondes represents a novel approach to Venus exploration on the scale of a New Frontiers mission.

\subsection{Scientific Objectives}

Stemming from the "follow the water" theme, the Thalassa mission concept features four scientific objectives that explore the role of water at Venus from the upper atmosphere to the surface. Ordered here from greatest to lowest altitude, those objectives are:

1) Characterize atmospheric water loss at Venus today, including the escape rates of $\mathrm{H}$ and $\mathrm{O}$, and the nature of the Venus atmosphere's interaction with the solar wind and plasma environment;

2) Determine the history of Venus' outgassing and water loss, including its $\mathrm{D} / \mathrm{H}$ ratio and noble gas concentrations, to gain new insight into the extent of outgassing of the planet's interior;

3) Search for current evidence of volcanically emitted water by mapping the spatial and temporal abundance of water in the lower atmosphere to ascertain whether outgassing occurs today; and

4) Document the role of water in the formation of tesserae to test whether this distinctive terrain type formed in the presence of abundant volumes of water and may be analogous to Earth's continents.

For space considerations, we do not include a science traceability matrix in this White Paper, but these scientific objectives are described in greater detail below.

\section{Science Objectives}

\subsection{Objective 1: Characterize Atmospheric Water Loss at Venus Today}

The deuterium-hydrogen $(\mathrm{D} / \mathrm{H})$ ratio in the atmosphere of Venus is about 100 times Earth's value (Donahue et al., 1982; Donahue and Hartle, 1992; Hartle et al., 1996). This enrichment in deuterium can be interpreted as either the signature of a lost primordial ocean of at least $0.3 \%$ the volume of a terrestrial ocean, or of a steady state in which water is continuously supplied to the surface of Venus by comets or volcanic outgassing, balancing loss through hydrogen escape (Grinspoon et al., 1993). The extreme aridity of Venus' atmosphere (with a water vapor content of $0.002 \%$ ) compared with Earth led numerous investigators to favor the first interpretation, i.e., that large amounts of water have likely been lost from Venus since its formation (e.g., Donahue et al., 1982; Grinspoon et al., 1993; Gillmann et al., 2009; Way and Del Genio, 2020).

Thermal escape to space, including hydrodynamic escape-whereby solar heating leads to the expansion of the atmosphere and escape of upper-atmospheric species-is regarded as an important process for the early atmosphere of Venus (Persson et al., 2018), although not to a large extent today 
(Kasting and Pollack, 1983). Low thermospheric temperatures ( $275 \mathrm{~K}$ daytime and $100 \mathrm{~K}$ nighttime) and a surface gravitational acceleration of $\sim 0.9 \mathrm{~g}$ mean that thermal escape of $\mathrm{H}$ and $\mathrm{O}$ is negligible today. Both $\mathrm{O}$ and $\mathrm{H}$ ions escape via several likely interconnected plasma processes driven by the solar wind. Non-thermal neutral $\mathrm{H}$ escape is controlled by photochemistry, whereas non-thermal neutral O escape results from sputtering by pickup $\mathrm{O}^{+}$ions (Donahue et al., 1997; Lammer et al., 2006; Kallio et al., 2011).

Transport of $O$ through the Venus upper atmosphere is also poorly understood. Airglow in the mesosphere (65-120 km altitude), including from $\mathrm{O}, \mathrm{O}_{2}$, and $\mathrm{OH}$, but especially the $\mathrm{NO}$ nightglow, serves to trace the atmospheric circulation in the transition zone between the super-rotation regime and the subsolar/anti-solar circulation at altitudes above $100 \mathrm{~km}$ (Gérard et al., 2017). Yet differences observed between the brightness peaks in local time of the $\mathrm{O}_{2}$ and $\mathrm{NO}$ nightglows, for example, illustrate our lack of knowledge of this region of the Venus atmosphere.

Major uncertainties therefore remain about atmospheric loss mechanisms at Venus. Acquiring well designed and continuous observations of the Venus upper atmosphere will provide vital information on the rates at which $\mathrm{H}$ and $\mathrm{O}$, and thus

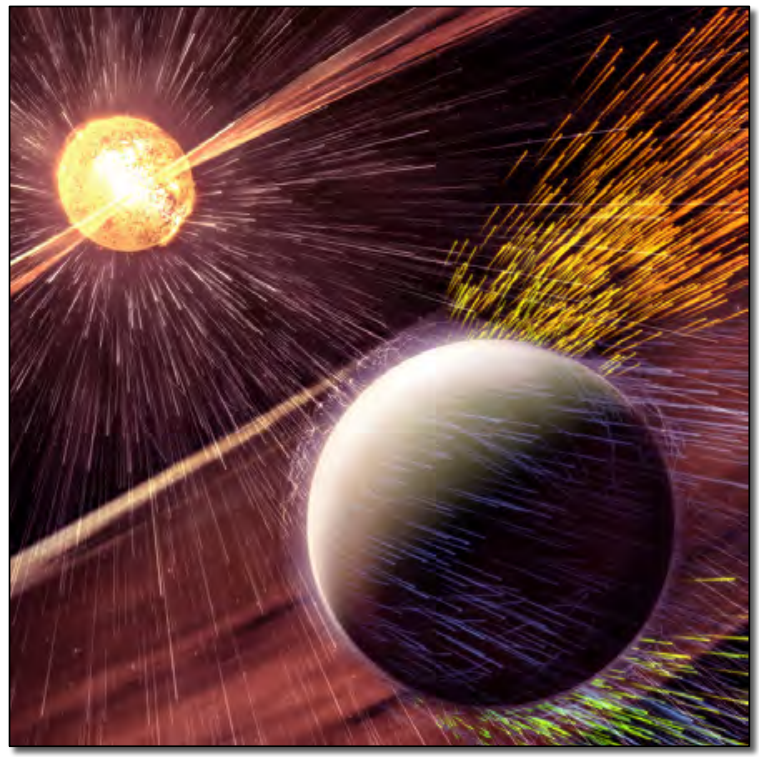

Figure 1. Thalassa will focus on characterizing atmospheric loss mechanisms at Venus to a precision not yet a chieved for the second planet. water, are lost to space at present (Figure 7). Such observations will contribute to a comprehensive understanding of how hydrogen and oxygen atoms are transported through the bulk of the atmosphere before escaping from the upper atmosphere, and will act as invaluable constraints for global circulation models.

Instrumentation: An orbital payload similar to that flown on NASA's Mars Atmosphere and Volatile Evolution (MAVEN) mission (Jakosky et al., 2018; Krasnopolsky, 2019) will effectively address this science objective. The MAVEN Neutral Gas and Ion Mass Spectrometer (NGIMS), coupled with the MAVEN Supra-Thermal And Thermal Ion Composition (STATIC) instrument (e.g., Jakosky et al., 2015), can assay the atmospheric escape of species such as $\mathrm{H}_{1} \mathrm{H}^{+}, \mathrm{He}^{+}, \mathrm{O}$, and $\mathrm{O}^{+}$. At lower altitudes in the mesosphere, the MAVEN Imaging Ultraviolet Spectrometer (IUVS) will allow for observation of NO nightglow (McClintock et al., 2015). IUVS will also permit extensive observations of the hydrogen and deuterium Lyman-alpha corona (Chaufray et al., 2012; Mayyasi et al., 2017). Since the MAVEN payload has been successfully operating at Mars since 2014, the technology readiness levels (TRLs) of the relevant instruments we plan to fly at Venus are high, i.e., TRL $>6$.

\subsection{Objective 2: Determine the History of Venus' Outgassing and Water Loss}

The middle and lower atmosphere of Venus contains critical information on the past and current outgassing history of the planet. Determining whether liquid water was ever present on Venus can be in part answered by characterizing atmospheric species in the modern bulk atmosphere. By doing so, we can address many outstanding questions such as how much water the Venus interior once possessed, when these outgassing events occurred, and if and when outgassing and water loss ended.

Measuring isotopic ratios of atmospheric noble gases such as $\mathrm{He}, \mathrm{Ar}, \mathrm{Kr}$, and Xe will allow us to place constraints on the sources and sinks of the past atmosphere. The majority of these species have not been measured at Venus to the accuracy necessary to understand their role in planet formation 
history. Because these noble gases would have outgassed together with large reservoirs of water vapor, measuring their abundances will help us understand when and how much water was injected from the interior to the surface and atmosphere in the geological past. Because the bulk atmosphere below the homopause at $\sim 125 \mathrm{~km}$ altitude is relatively well mixed, in situ measurements taken anywhere below this altitude will be sufficient to determine the abundances of these noble gases and their isotopes (Figure 2). The D/H ratio at the cloud tops and above have been well sampled (e.g., Fedorova et al., 2008; Marcq et al., 2017), but the middle and lower atmosphere data have large uncertainties (e.g., Marcq et

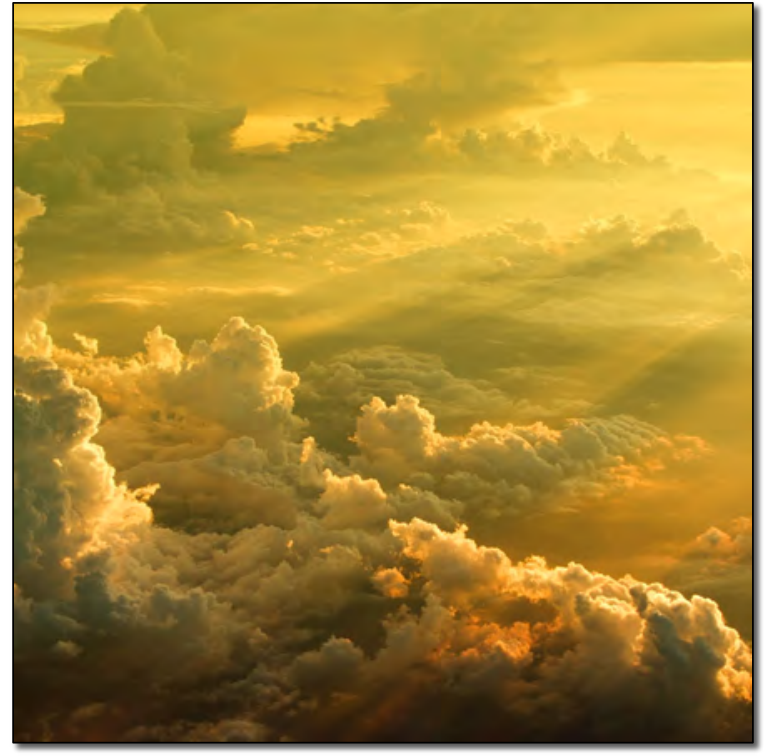

Figure 2. By returning middle-atmosphere composition data with unprecedented accuracy, Thalassa will establish Venus' degassing history. al., 2017; Johnson and de Oliveira, 2019). Better information on the variability of this ratio, especially near the surface, will substantially improve our understanding of Venus' primordial water content.

Instrumentation: A high-mass-resolution mass spectrometer is the key instrument for this objective. Two previous instrument designs can be considered for the Thalassa aerial platform: the Venus Mass Spectrometer (VMS) proposed for the DAVINCI Discovery mission (Glaze et al., 2016) and the Quadrupole Ion Trap Mass Spectrometer (QITMS) proposed for the Cupid's Arrow SmallSat mission, in turn a technology demonstration for the VERITAS Discovery mission (Bienstock et al., 2016). Both instruments are at TRL $\geq \mathbf{5}$ at present because VMS has heritage from the MSL Quadrupole Mass Spectrometer, and QITMS has undergone substantial development work at the Jet Propulsion Laboratory.

\subsection{Objective 3: Search for Current Evidence of Volcanically Emitted Water}

The Venus deep troposphere (from $\sim 15 \mathrm{~km}$ altitude to the surface) contains a minute abundance of water vapor ( 30 ppmv), and as yet no spatial or temporal variability in this abundance has been detected (Haus et al., 2015). On Earth, volcanic eruptions are a leading mechanism by which water vapor is injected into the atmosphere, with modern volcanic plumes containing large amounts of $\mathrm{H}_{2} \mathrm{O}$ (e.g., Burton et al., 2000). A volume of water typical of a volcanic plume on Earth will likely be easier to detect than on our own planet because of the low background variability of tropospheric water vapor abundances. By studying near-surface atmospheric water vapor concentrations, Thalassa will search for evidence of recent or ongoing volcanism, with the main objective of determining whether Venus's interior contains amounts of water comparable with modern Earth (Figure 3).

Although no mission has yet been dispatched to Venus with the dedicated purpose of searching for

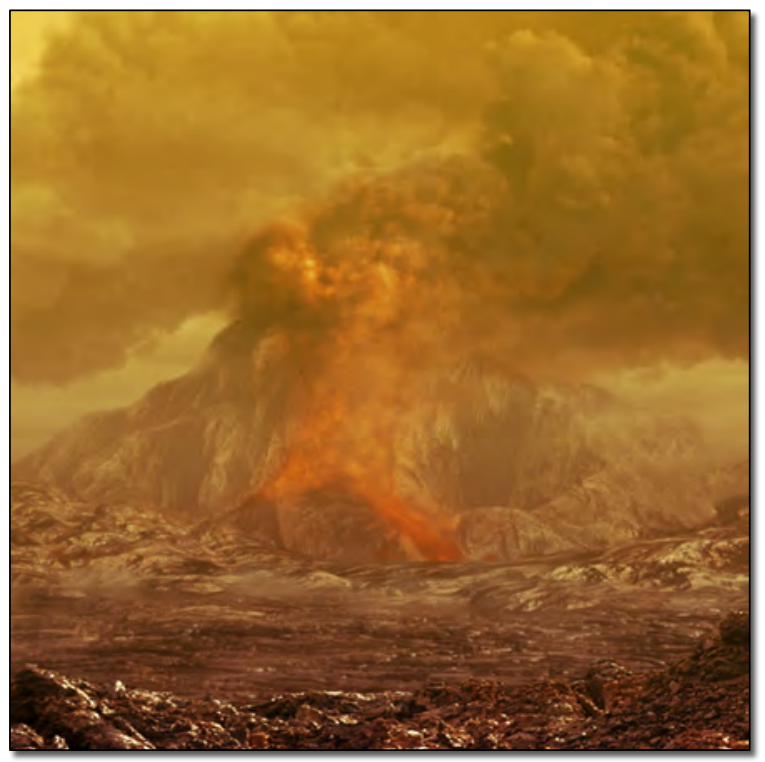

Figure 3. Thalassa will search for evidence of injections of water into the atmosphere, helping to answer whether Venus is volcanically active. 
modern volcanism, there is circumstantia/evidence that such activity is occurring today, or at least has taken place in the geologically recent past. For example, data from the Visible and Infrared Thermal Imaging Spectrometer (VIRTIS) on Venus Express (VEx) suggest that variations in surface emissivity in proximity to known volcanic edifices may correspond to differences in surface weathering and thus could denote relatively young lava flows (Smrekar et al., 2010), and the global $\mathrm{H}_{2} \mathrm{SO}_{4} \cdot \mathrm{H}_{2} \mathrm{O}$ cloud layer might be the result of volcanic outgassing over the past several tens of millions of years (e.g., Bullock and Grinspoon, 2001). The strong mesospheric variability in sulfur dioxide could reflect ongoing volcanic activity (Esposito et al., 1988), and the VEX Venus Monitoring Camera returned evidence for transient hot spots on the planet's surface consistent with recent lava flows (Shalygin et al., 2015). Even the nondetection of water vapor variability in the lower atmosphere by Thalassa would provide a key insight into the rate of atmospheric replenishment-and thus volcanism-at Venus.

Instrumentation: Repeated mapping observations in nightside near-infrared (NIR) window regions allows mapping of $\mathrm{H}_{2} \mathrm{O}$ and other potentially volcanogenic gases. A combination echelle-type slit spectrometer and grating spectrometer was flown as the VIRTIS instrument on VEx (Drossart et al., 2007). However, VIRTIS was not optimized for the specific science questions its team tried to answer. As a result, the Venus Emissivity Mapper (VEM) (Helbert et al., 2018) was developed for recent Venus exploration efforts such as the VERITAS Discovery mission (Smrekar et al., 2020) proposed to NASA, as well as the EnVision mission proposal (Ghail et al., 2018). Atmospheric $\mathrm{H}_{2} \mathrm{O}$ abundance at altitudes of O$15 \mathrm{~km}$ will be found by measuring from orbit the absorption band near the flank of the emission windows at 0.92 and $1.19 \mu \mathrm{m}$, using a VEM-based instrument; the VEM instrument itself is at systemslevel TRL 6 (Helbert et al., 2018).

\subsection{Objective 4: Document the Role of Water in the Formation of Tesserae}

Only radar data from the Magellan mission (Solomon et al., 1992; Phillips and Hansen, 1994), 1 $\mu \mathrm{m}$ emissivity data from Venus Express (Gilmore et al., 2015; 2017), and a handful of in situ analyses from landed missions (e.g., Treiman, 2007) are available to characterize surface rock type and mineralogy. The generation of partial melt from a source rock, together with the mixing of crustal materials and/or the introduction of water, can yield a range of rock compositions from "mafic" (iron-rich, silica-poor)

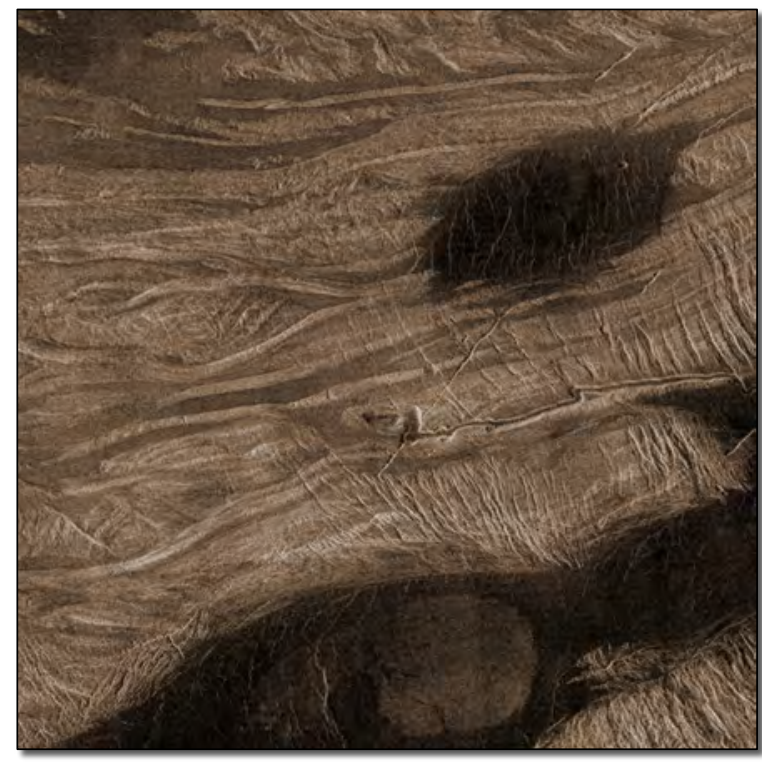

Figure 4. Did Venus' tessera ancient regions form in a manner similar to Earth's continental materials? Thalassa will tackle this question head on. basalt to "felsic" (iron-poor, silica-rich) rhyolite and its intrusive equivalent, granite-each with important implications for a planet's geological evolution.

This rock-type distinction is key to understanding the extent to which water has played a role in the geological history of Venus, and, in particular, to addressing the formation of tessera terrain, which occupies $\sim 7 \%$ of the planet's surface and is interpreted as the locally oldest material on Venus (Ivanov and Head, 2011) (Figure 4). Tesserae are tectonically complex units with styles of deformation not observed on any other planetary body (other than, perhaps, exposed continental interiors on Earth). Tesserae occur as large plateaus thousands of kilometers across and as smaller inliers: isolated, high-standing regions hundreds of kilometers in breadth that are embayed by younger volcanic deposits (Ivanov and Head, 2011). 
One interpretation is that the tesserae represent a thin layer of basalt that was the cooling crust of a massive subsurface magma pond (e.g., Hansen, 2006). Another view is that tesserae may be the Venus counterparts of continents on Earth, on the basis of morphology, gravity signature, and inferred composition (Hashimoto and Sugita, 2003; Hashimoto et al., 2008; Romeo and Capote, 2011). Indeed, data from Galileo and VEX together suggest that tesserae may be compositionally distinct from most of the rest of the Venus surface (Helbert et al., 2008; Mueller et al., 2008). For example, surface emissivity data from VIRTIS on VEX provide supporting evidence that the Alpha Regio tessera is more felsic than adjacent basaltic plains (Gilmore et al., 2017). Although fractional crystallization can yield felsic rocks from mafic (or ultramafic) source rocks, the high volume ratio of mafic to felsic melts would challenge such an origin for tessera terrain if the composition of the materials comprising tessera at the surface represented that of the full column of underlying crust (e.g., Gilmore et al., 2017). These arguments are consistent with the hypothesis that the tesserae are analogous to granitic continental materials on Earth, which form via crustal recycling in the presence of substantial volumes of subsurface water (e.g., Campbell and Taylor, 1983). Because felsic rocks have proportionately more silicon and less iron than silica-poor mafic rocks, characterizing the total iron abundances of Venus' tessera terrain offers a direct means to address whether these units formed in a period of abundant liquid water on Venus (Dyar et al., 2018).

Instrumentation: The VEM-based NIR instrument needed for Objective 3 (§2.3) will meet this objective. Yet, from orbit or at the cloud layer to $\sim 50 \mathrm{~km}$ altitude, we are restricted to assessing larger tessera units because scattering from Venus' persistent cloud deck limits spatial resolution. By collecting NIR spectra below the clouds, however, smaller tessera inliers can be resolved, strengthening the interpretation of global radar imagery and orbital NIR data for larger tesserae. Therefore, the aerial platform will also deploy a miniature version of the orbiter-hosted NIR spectrometer on dropsondes. As each dropsonde falls, more of the $\mathrm{CO}_{2}$ absorption window becomes transparent until, near the surface, a continual spectral band at 0.8-1.5 $\mu \mathrm{m}$, free of atmospheric absorption, becomes observable.

\section{Notional Mission Architecture}

The Thalassa mission architecture combines a number of elements with high flight heritage. For example, the orbiter leverages the tremendous success of the NASA MAVEN mission, which has been measuring the composition and loss rates of gases in the Martian atmosphere from orbit since 2014. An instrument suite based on MAVEN's heritage is proposed for Thalassa's orbiter element in order to characterize Venus' present $\mathrm{O}$ and $\mathrm{H}$ atmospheric loss rate (\$2.1).

The orbiter will also search for evidence of recent water injection into the lower Venus atmosphere (§2.3), and will determine the iron content of major tessera units across the surface (\$2.4). An aerial platform, taking inspiration from the Soviet Vega balloons that explored the Venus clouds in 1984-85, but incorporating recent and in-development capabilities (e.g., Cutts et al., 2018), will assay the gas and cloud composition of the middle Venus atmosphere (\$2.2). And a set of dropsondes, leveraging insight gained from the 2010 Venus Climate Mission concept study, will target a subset of the tesserae resolved by the orbiter (§2.4).

Our proposed combination of orbiter, aerial platform, and dropsondes represents a novel approach to Venus exploration on the scale of a New Frontiers mission. In addition to representing a compelling exploration target itself, Venus offers us a natural laboratory for learning how large rocky planets form and evolve-including planets in orbit about other stars, and even Earth itself.

So isn't it time we went back? 


\section{References}

Bienstock, B., Darrach, M., Madzunkov, S., and Sotin, C. (2015) Cupid's Arrow: An innovative nanosat to sample Venus' upper atmosphere. 2016 IEEE Aerospace Conference, Big Sky, Montana, March 5-12, 2016.

Bullock, M. A, and Grinspoon, D. H. (2001) The recent evolution of climate on Venus. Icarus, 150, 19-37.

Burton, M. R., Oppenheimer, C., Horrocks, L. A., and Francis, P. W. (2000) Remote sensing of $\mathrm{CO}_{2}$ and $\mathrm{H}_{2} \mathrm{O}$ emission rates from Masaya volcano, Nicaragua. Geology, 28, 915-918

Campbell, I. H., and S. R. Taylor. (1983) No water, no granites - No oceans, no continents. Geophys. Res. Lett. 10, 1061-1064.

Chaufray, J-Y., Bertaux, J-L, Quémerais, E., Villard, E., and Leblanc, F. (2012) Hydrogen density in the dayside venusian exosphere derived from Lyman- $\alpha$ observations by SPICAV on Venus Express. Icarus, 217, 767-778.

Cutts, J. A., and the Venus Aerial Platform Study Team (2018) Venus Aerial Platform Study. JPL D-102569, Jet Propulsion Laboratory, Pasadena, CA

Donahue, T. M., and Hartle, R. E. (1992). Solar cycle variations in $\mathrm{H}^{+}$and $\mathrm{D}^{+}$densities in the Venus ionosphere: Implications for escape. Geophys. Res. Lett., 19, 2449-2452.

Donahue, T. M., Hoffman, J. H., Hodges, R. R., Jr., and Watson, A. J. (1982) Venus was wet: A measurement of the ratio of deuterium to hydrogen. Science, 216, 630-633.

Drossart, P., et al. (2007) Scientific goals for the observation of Venus by VIRTIS on Venus Express mission. Planet. Space Sci., 55, 1653-1672.

Dyar, M. D., et al. (2018) Venus surface oxidation and weathering as viewed from orbit with six-window VNIR spectroscopy. 16th VEXAG Meeting 2018, LPI Contributions 2137, abstract 8015

Elkins-Tanton, L. T., Smrekar, S. E., Hess, P. C., and Parmentier, E. M. (2007) Volcanism and volatile recycling on a one-plate planet: Applications to Venus. J. Geophys. Res., 112, E04S06, doi:10.1029/2006JE002793.

Esposito, L. W., Copley, M., Eckert, R., Gates, L., Stewart, A. I. F., and Worden, H. (1988) Sulfur dioxide at the Venus cloud tops, 1978-1986. J. Geophys. Res., 93, 5267-5276

Fedorova, A., et al. (2008) $\mathrm{HDO}$ and $\mathrm{H}_{2} \mathrm{O}$ vertical distributions and isotopic ratio in the Venus mesosphere by Solar Occultation at Infrared spectrometer on board Venus Express. J. Geophys. Res., 113, E00B22, doi:10.1029/2008JE003146.

Gérard, J.-C., et al. (2017) Aeronomy of the Venus upper atmosphere. Space Sci. Rev., 212, 1617-1683.

Ghail, R. C., et al. (2018) VenSAR on EnVision: Taking earth observation radar to Venus. Int. J. Appl. Earth Obs. Geoinf., 64, 365-376.

Gillmann, C. Chassefière, E. and Lognonné, P. (2009) A consistent picture of early hydrodynamic escape of Venus atmosphere explaining present $\mathrm{Ne}$ and $\mathrm{Ar}$ isotopic ratios and low oxygen atmospheric content. Earth Planet. Sci. Lett., 286, 503-513.

Gilmore, M. S., Mueller, N., and Helbert, J. (2015) VIRTIS emissivity of Alpha Regio, Venus, with implications for tessera composition. Icarus, $254,350-361$.

Gilmore, M. S., Treiman, A., Helbert, J., and Smrekar, S. (2017) Venus surface composition constrained by observation and experiment. Space Sci. Rev., 212, 1511-1540.

Glaze, L. S., et al. (2016) DAVINCI: Deep Atmosphere Venus Investigation of Noble Gases, Chemistry, and Imaging. Lunar Planet. Sci., 47, abstract 1560 .

Grinspoon, D. H., et al. (1993) Probing Venus's cloud structure with Galileo NIMS. Planet. Space Sci., 41, 515-542.

Hansen, V. L. (2006) Geologic constraints on crustal plateau surface histories, Venus: The lava pond and bolide impact hypotheses. J. Geophys. Res., 111, E11010, doi:10.1029/2006JE002714

Hartle, R. E., Donahue, T. M., Grebowsky, J. M., and Mayr, H. G. (1996) Hydrogen and deuterium in the thermosphere of Venus: Solar cycle variations and escape. J. Geophys. Res. Planet., 101, 4525-4538.

Hashimoto, G. L., and Sugita, S. (2003) On observing the compositional variability of the surface of Venus using nightside near-infrared thermal radiation. J. Geophys. Res., 108, doi:10.1029/2003JE002082

Hashimoto, G. L., et al. (2008) Felsic highland crust on Venus suggested by Galileo Near-Infrared Mapping Spectrometer data. J. Geophys. Res. Planet., 113, EOOB24, doi:10.1029/2008JE003134.

Haus, R., Kappel, D., and Arnold, G. (2015) Lower atmosphere minor gas abundances as retrieved from Venus Express VIRTIS-M-IR data at 2.3 4 m. Planet. Space Sci., 105, 159-174.

Helbert, J., Müller, N., Kostama, P., Marinangeli, L. Piccioni, G., and Drossart. P. (2008) Surface brightness variations seen by VIRTIS on Venus Express and implications for the evolution of the Lada Terra region, Venus. Geophys. Res. Lett. 35, doi:10.1029/2008GLO33609.11.

Helbert, J., et al. (2018) The Venus Emissivity Mapper (VEM): Obtaining global mineralogy of Venus from orbit. Proc. SPIE 10765, Infrared Remote Sensing and Instrumentation XXVI, 707650D

Ivanov, M. A., and Head, J. W. (2011) Global geological map of Venus. Planet. Space Sci., 59, 1559-1600.

Jakosky, B. M. (2018) Loss of the Martian atmosphere to space: Present-day loss rates determined from MAVEN observations and integrated loss through time. Icarus, 315, 146-157.

Jakosky, B. M., et al. (2015) MAVEN observations of the response of Mars to an interplanetary coronal mass ejection. Science, 350, aad0210, doi:10.1126/science.aad0210.

Johnson, N. M., and de Oliveira, M. R. R. (2019) Venus atmospheric composition and in situ data: A compilation. Earth Space Sci., 6, 1299-1318.

Kallio, E, Chaufray, J-Y Modolo, R Snowden, D, and Winglee, R (2017) Modeling of Venus, Mars, and Titan Space Sci Rev, 162, 267-307.

Kasting, J. F., and Pollack, J. B. (1983) Loss of water from Venus. I. Hydrodynamic escape of hydrogen. Icarus, 53, 479-508.

Krasnopolsky, V. A. (2019) Spectroscopy and photochemistry of planetary atmospheres and ionospheres: Mars, Venus, Titan, Triton and Pluto. Cambridge University Press, pp. 556

Lammer, H., et al. (2006) Loss of hydrogen and oxygen from the upper atmosphere of Venus. Planet. Space Sci., 54, 1445-1456.

Marcq, E., Mills, F. P., Parkinson, C. D., and Vandaele, A. C. (2018) Composition and chemistry of the neutral atmosphere of Venus. Space Sci. Rev., $214: 10$.

Mayyasi, M., et al. (2017) The variability of atmospheric deuterium brightness at Mars: Evidence for seasonal dependence. J. Geophys. Res. Space Phys., 122, 10,811-10,823.

McClintock, W. E et al. (2015) The imaging ultraviolet spectrograph (IUVS) for the MAVEN mission Space Sci. Rev 195, 75-124.

Mueller, N., Helbert, J., Hashimoto, G. L., Tsang, C. C., Erard, S., Piccioni, G., and Drossart, P. (2008) Venus surface thermal emission at 1 um in VIRTIS imaging observations: Evidence for variation of crust and mantle differentiation conditions. J. Geophys. Res., 113, doi:10.1029/2008JE003118. E5.

Persson, M., Futaana, Y., Federov, A., Nilsson, H., Hamrin, M., and Barabash, S. (2018) $\mathrm{H}^{+} / \mathrm{O}^{+}$escape rate ratio in the Venus magnetotail and its dependence on the solar cycle. Geophys. Res, Lett., 45, 10,805-10,811.

Phillips, R. J., and Hansen, V. L. (1994) Tectonic and magmatic evolution of Venus. Ann. Rev. Earth Planet. Sci., 22, 597-656.

Romeo, I., and Capote, R. (2011) Tectonic evolution of Ovda Regio: An example of highly deformed continental crust on Venus? Planet. Space Sci., $59,1428-1445$

Shalygin, E V., Markiewicz, W. J., Basilevsky, A. T., Titov, D. V., Ignatiev, N. I., and Head, J. W. (2015) Active volcanism on Venus in the Ganiki Chasma rift zone. Geophys. Res. Lett., 42, 4762-4769.

Smrekar, S. E., et al. (2007) Tectonic and thermal evolution of Venus and the role of volatiles: Implications for understanding the terrestrial planets. In Venus as a Terrestrial Planet (eds. Esposito, L. W., Stofan, E. R., and Cravens, T. E.). Washington, DC: American Geophysical Union Geophysical Monograph Series, 176, pp. 45-71.

Smrekar, S. E., et al. (2010) Recent hotspot volcanism on Venus from VIRTIS emissivity data. Science, 328, 605-608.

Smrekar. S. E., et al. (2020) VERITAS (Venus Emissivity, Radio Science, InSAR, Topography And Spectroscopy): A proposed Discovery mission. Lunar Planet. Sci., 51, abstract 1449

Solomon, S. C., et al. (1992) Venus tectonics: An overview of Magellan observations. J. Geophys. Res., 97, 13,199-13,255.

Tosi, F., et al. (2017) Temperature-dependent VNIR spectroscopy of hydrated Na-carbonates. 19th EGU General Assembly, abstract 7375.

Treiman, A. H. (2007) Geochemistry of Venus' surface: Current limitations as future opportunities. In Venus as a Terrestrial Planet (eds. Esposito, L. W., Stofan, E. R., and Cravens, T. E.). Washington, DC: American Geophysical Union Geophysical Monograph Series, 176, pp. 7-22.

Way, M. J., and Del Genio, A. D. (2020) Venusian habitable climate scenarios: Modeling Venus through time and applications to slowly rotating Venus-like exoplanets. J. Geophys. Res. Planet., 125, e2019JE006276. 